\title{
Meta
}

Journal des traducteurs

Translators' Journal

\section{Studies of Translation Models 3 : An Interaction Model of the Translation Process}

\section{Ronald H. Bathgate}

Volume 30, numéro 2, juin 1985

URI : https://id.erudit.org/iderudit/002000ar

DOI : https://doi.org/10.7202/002000ar

Aller au sommaire du numéro

Éditeur(s)

Les Presses de l'Université de Montréal

ISSN

0026-0452 (imprimé)

1492-1421 (numérique)

Découvrir la revue

Citer cet article

Bathgate, R. H. (1985). Studies of Translation Models 3 : An Interaction Model of the Translation Process. Meta, 30(2), 129-138. https://doi.org/10.7202/002000ar
Résumé de l'article

A graphical notation permitting a detailed description of the translation process is developed and used to describe a number of different translation situations. This notation, based on a recently published model of the translation process, lays particular emphasis on the interactive nature of the translation process, i.e. on the fact that a translator does not work in isolation but is a member of a team whose task is to produce an optimum translation by joint effort. A written notation equivalent to the graphical notation is also briefly introduced. 


\title{
STUDIES OF TRANSLATION MODELS 3 AN INTERACTIVE MODEL OF THE TRANSLATION PROCESS
}

RONALD H. BATHGATE

\begin{abstract}
A graphical notation permitting a detailed description of the translation process is developed and used to describe a number of different translation situations. This notation, based on a recently published model of the translation process, lays particular emphasis on the interactive nature of the translation process, i.e. on the fact that a translator does not work in isolation but is a member of a team whose task is to produce an optimum translation by joint effort. $A$ written notation equivalent to the graphical notation is also briefly introduced.
\end{abstract}

\section{INTRODUCTION}

So far, models of the translation process have been made on the assumption that a translation is something made by a translator. In general, however, this is only part of the truth. A translation is often, and should in general be, something made by a translator together with one or more advisers - often his or her customer.

This fact is expressed in a proposed "operational" model of the translation process $(1,2,3)$ by including "discussion" as the final phase of the translation process (see Fig. 1). As mentioned in the above publications, this does not mean that the discussion phase is always restricted to the end of the translation process : it often overlaps with the tuning, understanding, terminology and possibly with the checking phase. Moreover, the discussion phase is the only one of the phases of the operational model which may be absent in a given translation operation.

For a detailed description of these various phases, see the previous publications mentioned.

In the present paper, we will develop this idea of translation as an interactive process with the aid of a graphical notation briefly introduced previously (see references).

\section{DEVELOPMENT OF THE GRAPHICAL NOTATION}

For the sake of convenience, we will start by developing our graphical notation with reference to the middle three phases of the operational model of Fig. 1 : understanding, terminology and restructuring.

\subsection{UNDERSTANDING}

The ability to understand can be pictured as based on the construction of a mental framework into which we can fit the elementary facts coming to our attention. This framework would seem itself to be built up of previously acquired facts : we understand the facts stated in a given text because we know more than is stated there. 
This image of the process of understanding is illustrated in Fig. 2. It may be compared with the input of data into a computer : the data come in a string (punched tape, magnetic tape, or a sentence) ; you take each element in turn, and fit it into the appropriate place in the framework (the computer memory, or the human understanding). The work of isolating the individual elements and assigning them to the right place in the framework is shown in Fig. 2 as being performed by an "input analyser" - which can be regarded as embodying the analysis phase of the operational model of Fig. 1.

\subsection{TERMINOLOGY}

To continue our computer analogy, the terminology phase can be regarded as the first stage of the processing of the data stored in the understanding framework.

It isn't enough for the translator to be able to understand the facts presented to him in the source language text $:$ he also has to be able to express them in the correct terminology. (I am assuming, throughout most of this article, that the translation to be made concerns a specialized technical subject. As any translator knows, the problems encountered in such a translation tend to differ widely from those met with in a general or literary translation.)

The changes in the text involved in transforming the language from common everyday language to specialized terminology are so far-reaching that it would often seem as if a specialized text (e.g. in the field of chemistry, or law) is dealing with a completely different world from that which we are accustomed to see around us. The notation developed here therefore represents the terminology phase as involving a change of framework, from the square lattice of the understanding framework to the diagonal (diamond) lattice of the terminology framework.

Since the semantic units of the source language text have to fit into the "understanding" grid, we denote them by squares in our graphical notation; similarly, since the semantic units from the "terminology" grid end up in the target language text, we represent that text by a sequence of diamonds.

One topic which the operational model leaves rather vague is the point at which the transfer from source language to target language accurs. In practice, the skilled translator will doubtless have achieved a great deal of this transfer as early as the understanding phase : by the time he has stored the facts presented by the text in his understanding framework, he will automatically have found the target language equivalent for them. I sometimes imagine this to occur by means of a card-in-slot mechanism : each element in the understanding network can be thought of as a coded slot, and when the "card" for the source language semantic element in question is put into the right slot, the corresponding target language card automatically drops out.

In more difficult cases, the transfer to the target language may not be complete until the restructuring phase has been reached.

However, in any case we may assume that the output of the terminology phase will be in the target language : while the translator should be well acquainted with the source language technical terms for the specialism in question, what he will be looking for during the terminology phase will be the target language technical terms. It is thus quite appropriate that the change of framework associated with the use of specialized terminology should coincide in our model with the change of semantic elements from source language to target language.

\subsection{RESTRUCTURING}

In terms of the present notation, the restructuring phase of the operational model can be regarded as more or less the opposite of the understanding phase : the individual facts stored in the terminology framework are taken out one by one, adapted if neces- 
sary to ensure that they fit together neatly, and strung together in the target language sentence. This is assumed to be done by an "output synthesizer" in the present model. The necessary adaptation of the semantic elements to form the final message is not represented explicitly in the model.

\subsection{TUNING}

We are now in a position to extend our graphic notation to the other phases of the operational model. Let us start with the tuning phase.

It seems intuitively reasonable to assume that we have a separate portion of our framework of understanding, and of our terminology framework, for each specialized subject with which we are acquainted. We may imagine there to be a "chemistry framework", a "law framework", and "electronics framework", etc. We may perhaps also assume the presence of a separate input analyser for each specialism.

Now one of the things we are doing in the tuning phase is activating the input analyser, understanding framework and terminology framework appropriate to the text to be translated, and suppressing the others. When we translate a given specialized text, we are only working actively with a relatively small part of our linguistic and semantic resources. This is represented in Fig. 3 by operation of a selector switch.

It may be noted that our graphical notation has been simplified in Fig. 3. The understanding framework is now represented by a large single square, and the terminology framework by a large single diamond. If the semantic elements from the source language sentence have been assigned to the appropriate slots in the framework, this is indicated by placing a black square (or diamond) in it. Fig. 3 thus illustrates various stages in the translation of a legal text : the tuning selector has routed the input to the legal input analyser, and the facts stated in the text have been stored in the appropriate places in the legal understanding and terminology networks. The linguistic and semantic resources for dealing with chemical texts, etc., remain unused.

Another activity which may occur during the tuning phase is expansion of the understanding or terminology framework, by background reading, discussion with an adviser, etc. In general, translators suffer from a lack of specialized technical knowledge. This will be indicated in our notation by shading the bottom part of the square or diamond in question. The size of the shaded portion will give an indication of the extent of the ignorance. The translator's customer or adviser, on the other hand, will have a great deal of specialized technical knowledge - and may well know more technical terms in the target language than the translators ; but he will tend to lack linguistic skills - otherwise there would be no need for the translator's services. This will be indicated by shading the top part of the framework.

\subsection{THE CHOICE BETWEEN TRANSLATING FOR PUBLICATION AND FOR INFORMATION}

It is appropriate at this point to pause to consider the two main situations which the translator may encounter in the translation of technical texts. In general, he will be translating either because the customer cannot read the source language text, or because the customer has some ideas in his head he would like to get down on paper in the target language, but lacks the necessary linguistic skills. In the former case, the target language text may be almost irrelevant : what the customer wants may be merely to get the facts expressed in the text into his head. In practice, the translator may then be asked to take all kinds of short-cuts, from the production of a rough translation without polishing (I remember one colleague of mine saying that the last $1 \%$ of polish on a translation takes $99 \%$ of the time !) via the production of a short written précis of the text to just reading the text through and telling the customer the essence of the main 
points it contains. These last two alternatives are generally much more difficult than the customer realizes, owing to the translator's lack of specialized technical knowledge, and many translators have to refuse such requests.

In terms of the model developed in this paper, we may say that in translation for information, the customer tends to lack the input analyser for the source language in question. He will generally have a perfectly functioning output synthesizer, however, since the target language is usually his mother tongue. (Though it does quite often happen that a translator translates e.g. into English to provide e.g. a Dutch reader with information, if the translator cannot write Dutch and his customer can read English even though it is not his mother tongue.)

It is relevant in this connection to assume that the output synthesizer is reversible, i.e. it can also act as an input analyser from the target language to the understanding and terminology frameworks. Similarly, the input analyser can be regarded as capable of acting as an output synthesizer from this understanding and terminology frameworks to the source language.

In the case of translation for publication, the customer will have an input analyser which works perfectly (in both directions), but his output synthesizer will be faulty for the production of texts in the target language. In general, it will be much better in the reverse direction, for the reading of such texts. In this case, the source language text is rather irrelevant : the basic problem is to get from the ideas in the customer's head to the corresponding target language text. It often happens in my experience that e.g. Dutch authors who write English fluently but with a lot of mistakes will write their text in English instead of Dutch, and ask the translator to correct it. However, the distortion introduced by their faulty output synthesizer is often so great that it would be simpler, and yield a better final result, if they were to write the original text in their mother tongue after all.

\subsection{CHECKING}

The checking phase can now be represented as follows. The translator takes the target language text, and runs it backwards through the output synthesizer (which thus acts as an input analyser) to regenerate the appropriate patterns in the understanding and terminology frameworks. These patterns are compared with standard patterns stored in the memory, to show up any discrepancies ("That isn't what the author really wanted to say", "that's spelt wrongly", or "that's the wrong technical term".) A "correction signal" is then fed forwards through the output synthesizer, to modify the target language text appropriately.

The checking phase thus constitutes use of a feedback loop, which as readers with any acquaintance with control engineering will know is the usual means employed for regulating the operation of any active system. It is thus appropriate that such feedback loops should be found in the translation process, to embody the degree of control we know to exist there.

It very often happens that the customer or another technical expert with a fair knowledge of the target language checks the translation for (mainly technical) errors. However, there is then a very real risk that the faulty output synthesizer will then introduce linguistic errors into the final text, at the same time as technical errors are corrected. This is one of the main reasons why discussion between translator and customer (or some other technical adviser) is so important. From now on, we will denote the presence of linguistic flaws in an output synthesizer (or input analyser) by giving the block representing this unit a wavy line at the top. Similarly, the presence of technical flaws will be denoted by a wavy line at the bottom of the block. 
This convention can be extended to the source language and target language texts : we will denote linguistic errors in the text by making the line above the sequence of semantic elements wavy, and technical errors by making the bottom line wavy.

\subsection{DISCUSSION}

Discussion may be seen as the sharing of two incomplete understanding (or terminology) frameworks, so as ideally to improve both parties' knowledge of the subject concerned. If we assume that the discussion is associated with the checking phase, the process can then be indicated as shown in Fig. 4 (which is a modified representation of the way this interactive model was introduced in previous publications - see introduction).

The zigzag portion of the line in Fig. 4 leading from the target language text with technical errors (left) to the final target language text (right) indicates that errors are removed by an iterative process in which improvements suggested either by the translator or the customer are successively discussed until a consensus is reached as to what is the best text. The "to and fro" of the discussion is represented by the zigzag line.

\section{APPLYING THE MODEL}

We are now in a position to apply the interactive model of the translation process developed above to a number of practical cases. We will start with that of translating for publication.

\subsection{TRANSLATING FOR PUBLICATION}

The procedure here is indicated in terms of the interactive model in Fig. 5. I would like to suggest that readers try to follow the "language" of this figure before turning to the "translation" into normal words which follows.

We may say that this process starts with a set of ideas in the mind (understanding and terminology frameworks) of the translator's customer, which he would like to put down on paper in the target language. Since, however, he lacks the linguistic skills for this, he composes a text in the source language (see Fig. 5, top left). The translator then translates the text (right-hand side of Fig. 5) to produce the first draft of this translation (marked by a subscript ${ }_{1}$ ). This text may be expected to contain some linguistic errors or imperfections due to oversights or to complex turns of phrase where more mature consideration suggests a better translation. These are indicated by the wavy line above the row of diamonds representing the target language text. The text will doubtless also contain some errors of substance ("technical errors") due to the translator's incomplete understanding of the subject matter of the text, indicated by the wavy line below the row of diamonds.

In general, the translator will now correct his own text (feedback loop leading to translation draft ${ }_{2}$ in Fig. 5) ; in this process, we may assume that his insights are sufficient to remove his own linguistic errors (the line above the row of diamonds becomes straight), but not his technical errors. It is in this state that the target language text is passed to the customer, who will generally check it as regards the success with which his ideas are put across. We assume that, as is often the case, the customer has a fair passive command of the target language, though as we have mentioned his active command of the language is faulty ; this is indicated by a wavy line at the top of the box representing the customer's output synthesizer (here working initially in reverse, an a input analyser for the target language). This correction loop gives rise to draft ${ }_{3}$ of the translation. We may assume that the technical errors have been removed (straight line at bottom) - but the customer is likely to have introduced linguistic errors in the course of his correction (wavy line at top), which have to be removed before the target language 
text is ready for sending to the publishers. This is ideally done in a third correction round based on discussion between the translator and his customer (the zigzag line in this feedback loop represents the interchange of information and views between the two), which leads to translation draft ${ }_{4}$ - as perfect as humanly possible.

Apart from the graphic notation shown in Fig. 5, this whole process can also be represented in terms of a written notation, as follows :

$\dot{\mathrm{T}} \dot{U}_{\mathrm{c}} \mathrm{x}^{\mathrm{x}} \rightarrow \mathrm{s}^{\mathrm{x}}$

$s 1^{x} \times A \cup T S_{t x x} \rightarrow t l_{1 \times x}^{x}$

$\mathrm{tl}_{1} \mathrm{xx}_{\mathrm{xx}} \times \mathrm{S} \mathrm{T} \mathrm{U} \mathrm{U}_{\mathrm{txx}} \rightarrow \mathrm{tl}_{2} \mathrm{xx}$

$\mathrm{tl}_{2 \times \mathrm{xx}} \times \mathrm{S} \mathrm{T} \mathrm{U} \underset{\mathbf{c}}{\mathrm{xx}} \rightarrow \mathrm{tl}_{3} \mathrm{xx}$

$\mathrm{tl}_{3}{ }^{\mathrm{xx}} \mathrm{x}\left(\mathrm{S} \mathrm{T} \mathrm{U}_{\mathrm{t} \mathrm{xx}}+\mathrm{ST} \mathrm{U}_{\mathrm{c}}{ }^{\mathrm{xx}}\right)^{3} \rightarrow \mathrm{tl}_{4}+\mathrm{ST} \mathrm{U}_{\mathrm{t} x}+\mathrm{ST} \mathrm{U}_{\mathrm{c}}{ }^{\mathrm{x}}$,

where $\mathrm{sl}=$ source language text, $\mathrm{tl}=$ target language text, $\mathrm{A}, \mathrm{U}, \mathrm{T}$ and $\mathrm{S}$ represent operation of the input analyser, understanding stage, terminology stage and output synthesizer respectively ; subscript ${ }_{c}$ refers to the customer and subscript ${ }_{t}$ to the translator ; a dot above a stage (as in $\dot{T}$ ) represents the presence of sl-or tl-related information in the stage in question; one or two superscript crosses ( $\mathrm{x}$ or ${ }^{\mathrm{xx}}$ ) represent the presence of a small or large amount of linguistic error in the stage or text in question ; and one or two subscript crosses $\left({ }_{x}\right.$ or ${ }_{x x}$ ) represent a small or large amount of non-linguistic ("technical") error.

The sequential operation of a number of stages is represented by writing the corresponding symbols one after the other ; these operations affect either information ( ${ }^{\circ}$ ) or a sl or tl text (indicated by a multiplication sign " $x "$ ).

For example, $\dot{\mathrm{T}} \mathrm{UA}_{\mathrm{c}} \mathrm{x} \rightarrow \mathbf{s} \mathbf{s}^{\mathrm{x}}$ means that operation of the terminology and understanding stages and the input analyser of the customer (acting in reverse, and containing a certain amount of intrinsic linguistic error) produces a source language text embodying a certain amount of linguistic error; while $s l^{x} x$ A U T $S_{t x x} \rightarrow \operatorname{tl}_{1} x_{x x}$ means that operation of the translator's input analyser, understanding and terminology stages and output synthesizer (embodying a relatively large amount of intrinsic technical error) produces the first draft of the target language text, which embodies a small amount of linguistic error (corrected by the translator himself in the next phase) and a relatively large amount of technical error (which the translator is in general not capable of correcting on his own).

A related case is that of correction of a target language text written by a customer whose mother tongue is not the target language. The process involved bears a close formal resemblance to that of Fig. 5. We may sum up the differences and resemblances as follows. The source language text does not occur at all in this process, since the customer has enough confidence and fluency to write the text directly in the target language ( draft $_{1}$ ); this draft may be assumed to be free of technical errors, but to embody an appreciable amount of linguistic errors - often amounting to distortions. The execution of the first correction loop by the translator, leading to draft ${ }_{2}$, will thus generally be much more time-consuming than that of Fig. 5. In many cases, the work involved will be as much as that for translation of a source language text written by the author; the main advantages of this approach are thus often the practice the author receives in writing a target language text, and the gain in self-confidence and fluency which this entails. (Remember, fluency is by no means the same thing as correctness!)

The further processing of the corrected text is ideally as in Fig. 5. 


\subsection{TRANSLATING FOR INFORMATION}

In this case, the customer is not the author of the source language text, nor does he have enough passive command of the source language to read it. The translator therefore produces a target language text, which the customer can read (in many cases, the target language will be his mother tongue, though this need not always be so). Since this text is not needed for publication purposes, the elaborate correction procedure shown in Fig. 5 will not be needed.

A similar case - that of verbal translation for information - does not even involve a target language text ; the translator merely tells the customer what is written in (selected parts of) the text. The customer will often have enough technical understanding of the source language text to be able to indicate which parts he would like translated, even though he may not know the source language at all. For example, the first section of an article - often the introduction - or the last section (often the conclusions) might be expected to contain useful information, as might the text surrounding tables or graphs containing interesting numerical or graphical information. We may expect this verbal translation to involve a certain amount of discussion (interchange of information).

\section{CONCLUSIONS}

The "interactive" notation developed in this paper proves capable of giving a consistent, detailed description of the translation process. Its chief merit would seem to be the means it offers for visualizing the various phases of the translation process in an easily understandable, memorable way so as to bring out clearly a number of facets of this process, in particular those associated with its interactive nature, i.e. the fact that the translator (ideally) forms part of a team working together to produce an optimum translation. It is hoped that this notation may prove useful for detailed study of the translation process, aimed at improving the effectiveness of translation operations or translator training programmes, or the development of improved machine (computerized) translation systems. Its easily understandable, memorable nature may also make it highly suited for teaching purposes.

While the graphical notation facilitates the clear presentation of various facets of the translation process, it does not lend itself well to further mathematical (computeraided) manipulation. The written notation briefly introduced at the end of this paper would seem to be more suitable for this purpose. The "multiplication" involved in this written notation would seem in view of the nature of the present model to be some kind of matrix multiplication. So far, no attempts have been made to operationalize it as such. However, it is hoped that such operationalization, if successful, might permit construction of a computerized translation model that could a) give a realistic simulation of the translation process in a limited semantic field forming the basis for an "intelligent" translation model ; b) possibly provide a basis for improved machine translation.

One final comment may be made in the latter connection. A frequent criticism of machine translation (MT) systems is that a good final translation is so far never possible without (extensive) pre- and post-editing. The essence of this criticism would seem to be that it is a pity that MT systems are interactive : it would be preferable if we could give the system a source language text and let it do all the translation work on its own. Since however the main insight embodied in the present model of the translation process is that you can't expect a human translator to function optimally in a non-interactive system, it would seem unreasonable to expect an MT system to be able to do so. 


\section{REFERENCES}

BATHGATE, R.H. (1980) : "Studies of Translation Models 1. An Operational model of the Translation Process", Incorporated Linguist, 19, pp. 113-114.

BATHGATE, R.H. (1980) : "A Survey of Translation Theory", Philips Terminology Bulletin, 9, pp. 15-24.

BATHGATE, R.H. (1981) : "Studies of Translation Models 2. A Theoretical Framework", Incorporated Linguist, 20, pp. 10-16.

\section{FIGURE 1}

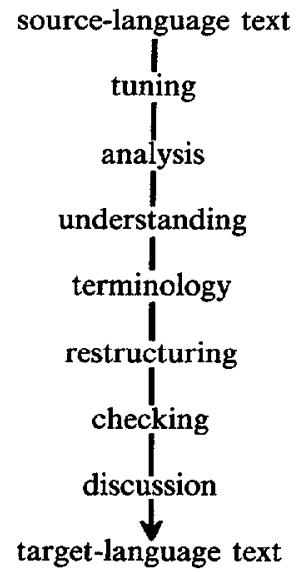

Fig. 1 : Operational model of the translation process.
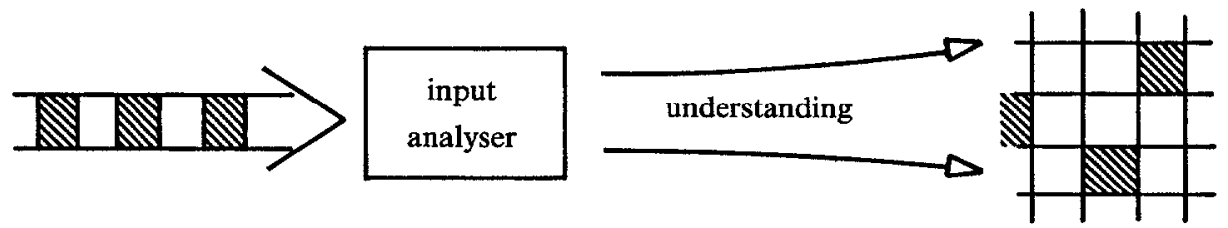

Fig. 2 : Understanding seen as pigeonholing data from a serial input string into a mental framework. 


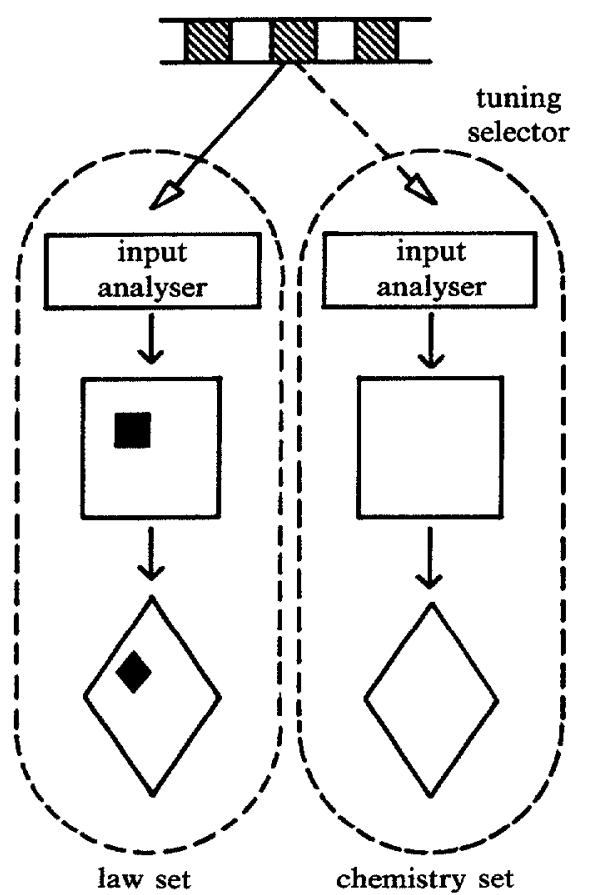

Fig. 3 : Tuning seen as selection of the appropriate set of linguistic and semantic resources for translation of a specialized text (a legal text in the present example). From now on, we simplify our representation of the "understanding" grid to a single square, and that of the terminology grid to a single diamond. If one of these grids contains information from (or destined for) the text to be processed, this is denoted by a small black square in the square, or a small black diamond in the diamond.

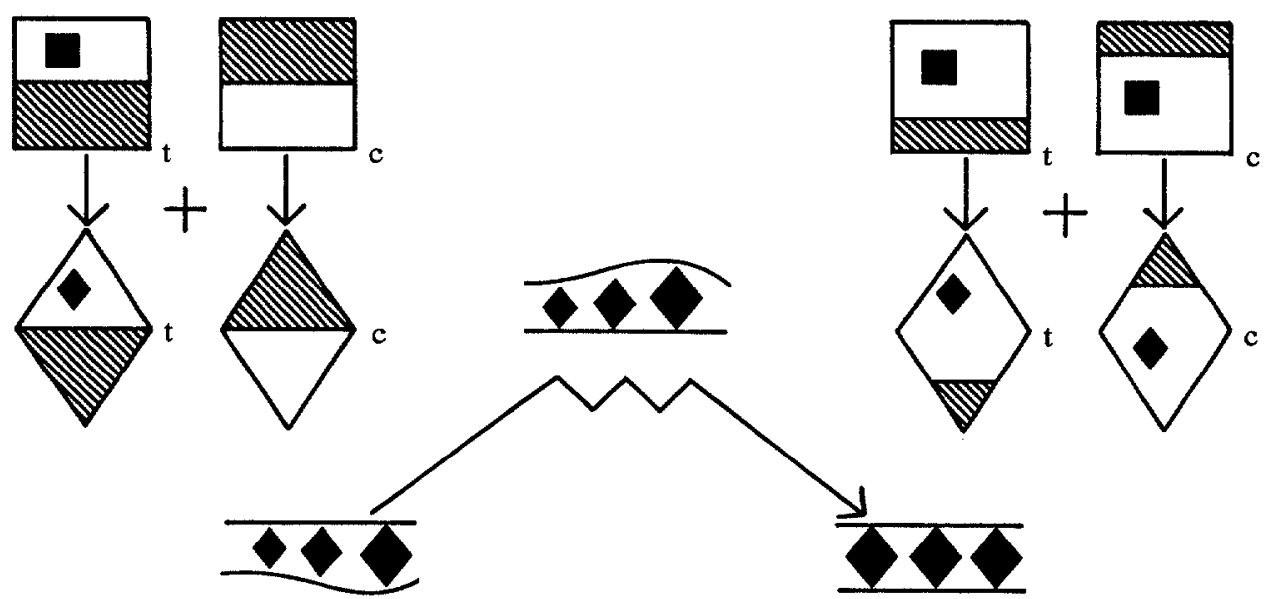

Fig. 4 : Discussion seen as sharing of incomplete understanding and terminology frameworks between translator and customer (or other technical adviser). By running through the feedback loop for correction several times together, they reduce the errors in the final target language text to a minimum and (ideally) learn in the process; the shaded areas of their understanding and terminology frameworks shrink. 


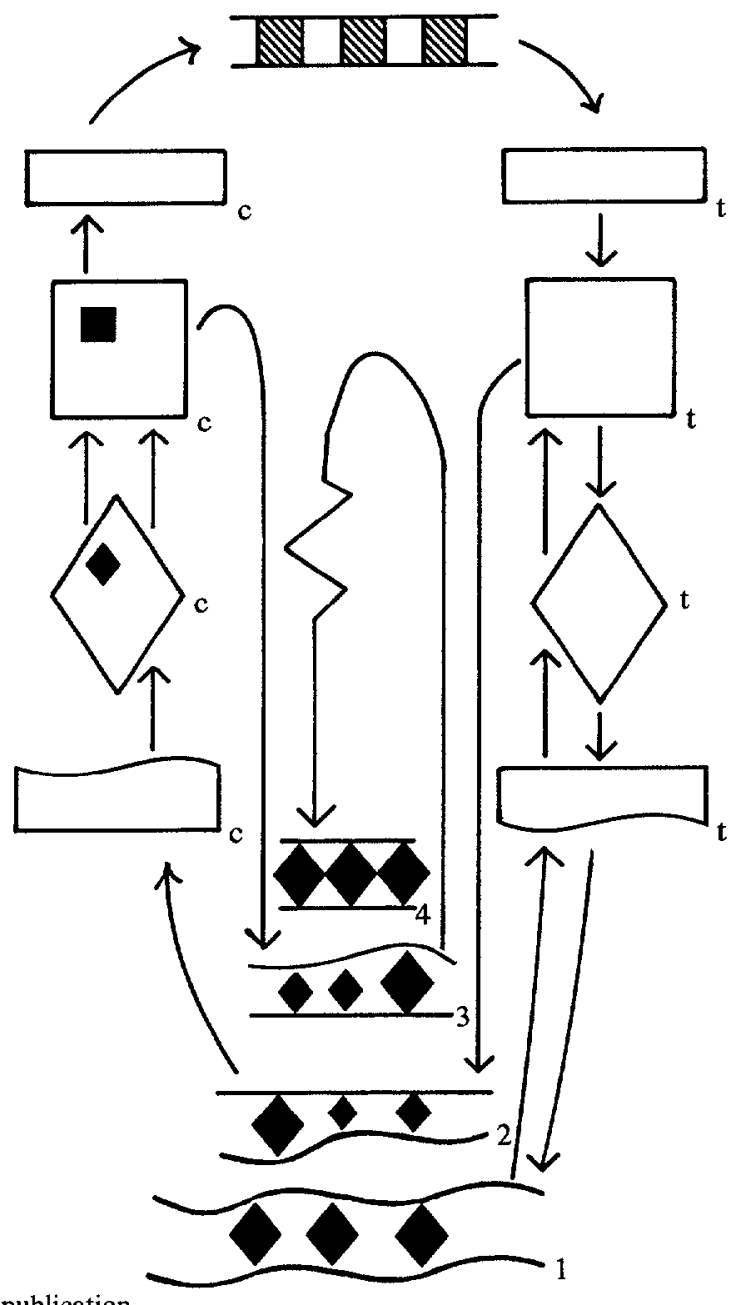

Fig. 5 : Translating for publication.

The sequence of events represented above can also be described in terms of a written notation, as explained in the text. 